\title{
Article
}

\section{Fullerton Virtual Twin Project: Overview and 2019 Update}

\author{
Nancy L. Segal and Francisca J. Niculae \\ Department of Psychology, California State University, Fullerton, CA, USA
}

\begin{abstract}
Virtual twins (VTs) are defined as same-age unrelated siblings raised together from early infancy. This special class of adoptive siblings replays the rearing situation of twins, absent genetic relatedness. The first such pair was identified and studied in 1990 at the University of Minnesota, leading to the creation of the Fullerton Virtual Twin Study (FVTS) at California State University, Fullerton (CSUF) the following year. The registry currently includes 169 VT pairs, mostly children, with new pairs identified on a regular basis. These sibling sets provide a direct estimate of environmental influences on developmental traits and, as such, offer informative comparisons with ordinary monozygotic and dizygotic twins, full siblings and adoptive brothers and sisters. The sample characteristics, assessment battery and findings to date are summarized in this 2019 update.
\end{abstract}

Keywords: Adoptees; evolutionary perspective; intelligence; virtual twins

(Received 21 April 2019; accepted 23 April 2019; First Published online 29 July 2019)

\section{An Overview of Virtual Twin Pairs}

Virtual twins (VTs) are a novel kinship, defined as same-age unrelated siblings who are reared together from early in infancy. Their unique quality is that they replicate twinship, but without the genetic link, thereby offering a direct estimate of how much environmental factors underlie variation in measured behavioral and physical traits (Segal, 2005, 2010, 2012, 2017). They are a more appropriate comparison with monozygotic (MZ) and dizygotic (DZ) twins, as well as siblings, than are pairs of ordinary adoptees because their age and residential histories are closely matched. In fact, VTs are the environmental counterparts of MZ twins reared apart who share $100 \%$ of their genes, but who are raised in different families. This article presents a full overview and update of the project since the 2013 registry report (Segal et al., 2013; also see http://drnancysegaltwins.org/virtual-twins-study).

The majority of VTs are classifiable into one of two main types: adoptive-adoptive or adoptive-biological. Infertile couples seeking adoption are sometimes offered two children at the same time or near in time. It is also possible that couples experiencing conception difficulties choose both reproductive assistance and adoption to improve their chances of having a family. Some, such couples conceive a child (or a multiple birth set) and are offered a same-age adoptee, also creating a VT pair. There are, in addition, other means by which VT sibling sets come into being. They include (1) sperm donation for maternal surrogacy, coupled with adoption; (2) unrelated embryo transfer, plus adoption; (3) natural conceptions by same-sex female partners, via different males and (4) twins switched at birth with an unrelated twin or nontwin infant, due to hospital oversight.

The Fullerton Virtual Twin Study (FVTS) includes examples of all of the above, the majority being adoptive-adoptive pairs. It is

Author for correspondence: Nancy L. Segal, Email: nsegal@fullerton.edu

Cite this article: Segal NL and Niculae FJ. (2019) Fullerton Virtual Twin Project: Overview and 2019 Update. Twin Research and Human Genetics 22: 731-734, https://doi.org/10.1017/ thg. 2019.40 worth noting that twins switched at birth and paired with an unrelated sibling form an unusual subset of VTs because they are raised believing that they are DZ twins (Segal \& Montoya, 2018). In contrast, the other VT sets are typically aware of their rearing status as unrelated siblings.

The FVTS currently includes 169 VT pairs, of which $106 \%$ or $63 \%$ are adoptive-adoptive and $63 \%$ or $37 \%$ are adoptive-biological. Opposite-sex and same-sex pairs occur with approximately equal frequency, as shown in Table 1 . The mean age of the siblings is 8.73 years $(S D=9.18)$, with a range of $4.01-54.84$ years. (Mean age is based on 325 individuals, following elimination of 13 siblings who are members of more than one pair.) The majority of individuals $(76.9 \%)$ are, however, younger than 8 years of age. The mean age difference between the $169 \mathrm{VT}$ sibling pairs is 3.06 months $(S D=2.72)$, with a range of $0-9.23$ months. The participants are identified on an ongoing basis, and there is interest in expanding the sample further.

\section{Goals and Directions}

The Fullerton VT Registry was designed with two major goals in mind. (1) The first is the estimation of how much shared environmental influences explain individual differences across a broad range of behavioral and physical characteristics. They include (but are not limited to) general intelligence, special mental abilities, personality traits, behavioral problems, height, weight, medical conditions and dentition. Comparative data from extant twin, sibling and adoption studies are used to evaluate the findings. (2) The second goal is to assess the nature of VT pairs' social relationships with one another and to compare them with those of $\mathrm{MZ}$ and DZ twins, peers and siblings. Given the varying degrees of genetic relatedness across these twin and sibling sets, applying an evolutionary framework adds depth and dimension to the interpretations. Related areas of interest include decision-making, peer associations and parenting. 
Table 1. Virtual twin pairs organized by pair type and sex composition

\begin{tabular}{lcccc}
\hline Pair type & MM & FF & MF & Total \\
\hline Adopted-adopted & 30 & 22 & 54 & 106 \\
\hline Adopted-biological & 19 & $16^{\text {a }}$ & 28 & 63 \\
\hline Total & 49 & 38 & 82 & 169 \\
\hline
\end{tabular}

$\mathrm{MM}=$ male-male, $\mathrm{FF}=$ female-female, $\mathrm{MF}=$ male-female

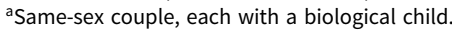

\section{Criteria for Study Participation}

Strict criteria for study participation have been in place since the inception of the study. Failure to fulfill even one requirement leads to disqualification. These criteria were generated so that VTs would match as closely to twins as possible; the rationale that guided these decision rules is provided in parentheses.

(1) Adoptive siblings must be placed in the family before 1 year of age. (Some co-twins are separated early in life due to differential prematurity, hospitalizations and other events, so early separation periods between co-VTs are acceptable.)

(2) The age difference between siblings must be 9 months or less. (Nine months is the maximum age difference between classmates.)

(3) School-age participants must be enrolled in the same grade. (Like twins, VT siblings may attend different classes or different schools but must be in the same grade.)

(4) Both siblings must be unaffected by birth trauma that may interfere with normal cognition. (The possibility that adverse birth events occurred is assessed by parental interview. Information is occasionally lacking or incomplete, requiring investigator judgement.)

(5) Participants must be at least 4 years of age to take part in the study. (Siblings of this age are generally comfortable with test situations and have formed social relationships with one another.).

VTs who differ in sex and/or ethnicity qualify for participation. Justification for their inclusion is that opposite-sex twins occur naturally and some ordinary DZ twins born to mixed-race couples show extreme physical differences (Segal, 2000).

\section{Recruitment and Procedures}

The majority of VTs are identified through publications and organizations (print and online) targeted to families with adopted children. Other sources include personal referrals, other research projects, the media and multiple birth organizations. Most VTs reside in the USA.

Following identification of a potentially qualified pair, the senior investigator contacts the family to review the specified criteria. At that time, if VTs are too young to participate, some preliminary forms are mailed to families to complete because some types of information (e.g., children's birth/adoption circumstances and early health history) are easily accessible when children are young.

A comprehensive packet of materials is mailed to families who qualify. Forms request information on children's birth and adoption histories, parental age, occupation and education, children's school history, physical facilities in the rearing home and other background measures. Parents also complete medical and dental forms, the Child Behavior Checklist (CBCL), the Adjective Checklist (ACL) for describing their children, the Index of Preadoption/Initial Adaptation to Adoption (Tan et al., 2010), a social relationship survey, the sibling inventory of differential experience, the Runco Ideational Behavior Scale (RIBS) that assesses creativity (O’Neal et al., 2015) and a Big Five personality questionnaire (Saucier \& Goldberg, 1998). Parents forward teacher versions of the CBCL and RIBS to their children's schools. VTs who are 18 years of age and older complete different versions of these forms on their own. The parents of these participants complete the same forms of the parents of the younger pairs.

A major part of the assessment is administration of the ageappropriate Wechsler Intelligence test to each sibling. With only a few exceptions, pair members are tested locally on the same day by separate examiners. All intelligence test protocols are reviewed for accuracy in scoring on receipt, allowing questions to be resolved with examiners prior to data entry and preparation of parental reports.

\section{Summary of Publications by Trait}

Findings from each analysis are summarized briefly according to area of study. The relevant FVTS findings follow, with complete citation details provided in the references section.

\section{General Intelligence (IQ Score)}

The most recent analysis ( $n=164$ pairs) yielded an intraclass correlation of $r_{i}=.25$ (Segal Li, Graham, Miller, \& McGuire, 2015). Thus, a modest contribution of the shared family environment to individual differences in IQ was indicated, consistent with earlier reports with fewer pairs. This sample included mostly young children, so it is likely that the magnitude of the correlation will decrease as the participants' age. This expectation is predicated on findings that unrelated siblings' IQ correlation decreases from .31 in childhood to .19 in adolescence (Scarr et al., 1993) and (based on a summary of the literature) from .25 in childhood to .00 in adulthood (McGue et al., 1993). It is, therefore, worth noting that an earlier analysis of $43 \mathrm{VT}$ pairs showed the expected decrease in IQ resemblance from .30 (age 5.11 years) to .11 (age 10.77 years; Segal et al., 2008).

\section{IQ Subtest Profile Analyses}

Profile analyses of the verbal and nonverbal IQ subtests compare samples of VTs, MZ twins and DZ twins. The VT correlation of $r_{i}=.11$ contrasted with the $r_{i}=.45$ for MZ twins and $r_{i}=.24$ for DZ twins, consistent with genetic effects (Segal \& Hershberger, 2005).

Additional papers and chapters reporting VT findings on general intelligence include Segal $(1997,2000)$, Segal and Hershberger (2005), Segal et al. (2007) and Segal et al. (2012).

\section{Nonverbal Cognition}

The Twin Testosterone Transfer Hypothesis has been referenced to explain the masculinization of some traits displayed by female twins with twin brothers (Tapp et al., 2011). However, because biological and social factors are confounded in these pairs, novel approaches are required. A unique design for disentangling these effects was possible by comparing the nonverbal cognition of females from dizygotic opposite-sex (DZOSf) pairs with females from virtual twin opposite-sex (VTOSf) pairs (Segal et al., 2019). The DZOSf twins showed a trend toward outperforming 
the younger VTOSf twins on the Block Design subtest of the Wechsler Intelligence test.

\section{Parenting}

Parental favoritism. Two competing hypotheses were tested using VTs, organized according to pair type, that is, adoptedadopted and adopted-biological: (1) Evolutionary theorizing predicts that parents should direct less favorable behaviors toward biologically unrelated children, relative to biologically related children, and express fewer favorable perceptions of unrelated children. This prediction is generated by kin selection theory, namely that altruistic acts should occur more often between genetic relatives than nonrelatives as a way of transmitting one's genes. (2) The compensatory model expects parents to favor biologically unrelated children over biologically related children. The reasoning behind this expectation is that parents strive to overcome what is viewed as an exceptional family structure. The VT sample allowed an informative test of these hypotheses, given that the children are age matched and share residential backgrounds. Parents did not distinguish between biological children and adoptive children on positive traits, but they did rate adoptive children higher on negative traits (Segal, Li, Graham, \& Miller, 2015).

Parental warmth. Parental warmth toward children was examined among parents and children using an eight-item scale, based on the acceptance-rejection subscale of the Children's Report of Parent Behavior Inventory (McGuire et al., 2012). Both genetic and nonshared environmental effects on children's reports were found. In contrast, parents' reports reflected both genetic and shared environmental influence.

\section{Adoption}

Developmental risks. Developmental risks across four content areas (Developmental Delays at Adoption, Initial Adaptation to Adoption, Crying/Clinging and Refusal/Avoidance), as well as age at adoption and various family factors, were compared between VTs and adopted together MZ Chinese twins. Both sibling sets share environments, but only the MZ twins share genes. Age at adoption contributed less than other predictors to adoptees' externalizing and internalizing behaviors, as measured by the CBCL. Genetic effects were evident across the four content areas, with shared environmental effects found for Developmental Delays at Adoption and Crying/Clinging. Family factors (e.g., parental education) contributed significantly to behavioral outcomes among the adopted Chinese twins (Segal et al., 2015).

\section{Social Behaviors and Within-Pair Relationships}

Tacit coordination. Tacit coordination (TC) refers to conditions in which 'two parties have identical interests and face the problem not of reconciling interests but only of coordinating their actions for their mutual benefit when communication is impossible' (Schelling, 1960, p. 54). MZ, DZ and VT pairs completed a TC task to assess the genetic influence on this behavior (Segal et al., 2008); a later paper compared MZ and DZ twins only (Segal et al., 2013). The measure of interest was the number of matched responses to questions answered under different experimental conditions. As predicted, MZ twins obtained the highest number of matches, followed by DZ twins and VTs. These results were consistent with both behavioral genetic and evolutionary psychological theorizing.
Friendships. Working independently, MZ twins, DZ twins, VTs, full siblings and friends listed the names of their friends, identifying those they had in common (McGuire \& Segal, 2013). Following this task, each pair discussed their answers and reached an agreement as to the total numbers of friends and shared friends. Hierarchical regression models identified dyad age, sex composition and genetic relatedness as significant predictors of peer overlap $(p<.001)$. MZ twins showed the highest percentage of peer overlap (82\%), while opposite-sex full siblings showed the lowest (27\%). Same-sex DZ twins (67\%) and VTs (62\%) showed relatively high agreement, in contrast with opposite-sex DZ twins (42\%) and VTs (37\%).

\section{Interpersonal Trust Beliefs}

The Children's Generalized Trust Belief Scale (Rotenberg et al., 2005) was used to examine interpersonal trust beliefs. MZ twins indicated significantly higher trust beliefs in their siblings than the other sibling types (McGuire et al., 2010). This finding agrees with evolutionary psychological expectations that interactants' genetic relatedness should affect within-pair social relatedness.

\section{Physical Traits}

Body mass index. The most recent analysis of body size was assessed by body mass index (BMI). The sample included 929 individuals, comprised of VTs, MZ twins and DZ twins from the Twins, Adoptees, Peers and Siblings Project, a collaboration between CSU Fullerton and the University of San Diego, and several other twin studies conducted at the University of Chicago, University of Minnesota and CSU Fullerton. Significant effects were found for nonadditive genetic and shared environmental influences. Specifically, $63.6 \%$ of the BMI variance was explained by a nonadditive genetic component, $25.7 \%$ by a common environmental component and $10.7 \%$ by an unshared component. It appears that both genetic and shared family factors (e.g., diet) contribute to individual differences in body size (Segal et al., 2008). Also in contrast with some previous studies, an earlier study found evidence of shared environmental effects (Segal \& Allison, 2002).

\section{Current and Future Directions}

Current and future projects include updating analyses of the IQ data, both overall score and subtest profile, as well as additional study of the CBCL and new analyses using the ACL. Fortunately, the availability of comparable data from young Chinese twins raised apart and together, collected at CSU Fullerton, will allow a series of novel comparative analyses (see e.g., Segal et al., 2011).

Acknowledgments. This work was supported by NIMH grant, R01 MH63351 (McGuire \& Segal), NSF awards BNS-7902583, BNS-8709207 and BNS9044080 (Segal) and several CSUF faculty research awards (Segal).

Contact Information. Please contact Dr Nancy L. Segal, Professor of Psychology and Director, Twin Studies Center, at California State University Fullerton, for participation or further information about the FVTS: nsegal@ fullerton.edu.

\section{References}

McGue, M., Bouchard, T. J., Jr., Iacono, W. G., \& Lykken, D. T. (1993). Behavioral genetics of cognitive ability: A lifespan perspective. In R. Plomin \& G. E. McClearn (Eds.), Nature, nurture and psychology (pp. 59-76). Washington, DC: APA Press. 
McGuire, S. A., \& Segal, N. L. (2013). Peers networks: Overlap in twin, sibling and friend dyads. Child Development, 84, 500-511.

McGuire, S. A., Segal, N. L., \& Hershberger, S. L. (2012). Parenting as phenotype: A behavior genetic approach to understanding parenting. Parenting: Science and Practice, 12, 192-201.

McGuire, S., Segal, N. L., Whitlow, B., Gill, P., \& Clausen, J. (2010). Sibling trust and trustworthiness. In K. J. Rotenberg (Ed.), Interpersonal trust during childhood and adolescence (pp. 133-154). Cambridge, England: Cambridge University Press.

O’Neal, I. C., Paek, S. H., \& Runco, M. A. (2015). Comparison of competing theories about ideation and creativity. Creativity: Theories - Research Applications, 2, 145-164.

Rotenberg, K.J., Fox, C., Green, S., Ruderman, L., Slater, K., Stevens, K., . . Carlo, G. (2005). Construction and validation of a children's interpersonal trust belief scale. British Journal of Developmental Psychology, 32, 271-292.

Saucier, G., \& Goldberg, L.R. (1998). What is beyond the Big Five? Journal of Personality, 66, 497-524.

Scarr, S., Weinberg, R. A., \& Waldman, D. (1993). IQ correlations in transracial adoptive families. Intelligence, 17, 541-555.

Schelling, T. C. (1960). The strategy of conflict. Cambridge, MA: Harvard University Press.

Segal, N. L. (1997). Same-age unrelated siblings: A unique test of within-family environmental influences on IQ similarity. Journal of Educational Psychology, 89, 381-390.

Segal, N. L. (2000). Entwined lives: Twins and what they tell us about human behavior. New York: Plume.

Segal, N. L. (2005). Indivisible by two: Lives of extraordinary twins. Cambridge, MA: Harvard University Press.

Segal, N. L. (2010). Twins: The finest natural experiment. Personality and Individual Differences, 49, 317-323.

Segal, N. L. (2012). Born together-reared apart: The landmark Minnesota twin study. Cambridge, MA: Harvard University Press.

Segal, N. L. (2017). Twin mythconceptions: False beliefs, fables, and facts about twins. San Diego, CA: Elsevier.

Segal, N. L., \& Allison, D. B. (2002). Twins and virtual twins: Bases of relative body weight revisited. International Journal of Obesity, 26, 437-441.

Segal, N. L., Feng, R., McGuire, A. S., Allison, D. B., \& Miller, S. (2008). Genetic and environmental contributions to body mass index: Comparative analysis of monozygotic twins, dizygotic twins and same-age unrelated siblings. International Journal of Obesity, 33, 37-41

Segal, N. L., \& Hershberger, S. L. (2005). Virtual twins and intelligence: Updated and new analyses of within-family environmental influences. Personality and Individual Differences, 39, 1061-1073.
Segal, N. L., Li, N., Graham, J. L., Miller, S. A., \& McGuire, S. A. (2015) Virtual twins: Individual differences and evolutionary perspectives. Paper presented at the International Society for the Study of Individual Differences, London, Ontario, Canada.

Segal, N. L., Li, N. P., Graham, J. L., \& Miller, S. A. (2015). Do parents favor their adoptive or biological children? Predictions from kin selection and compensatory models. Evolution and Human Behavior, 36, 379-388.

Segal, N. L., McGuire, S. A., Graham, J. L., \& Stohs, J. H. (2013). Fullerton Virtual Twin Study: An update. Twin Research and Human Genetics, 16, 451-454.

Segal, N. L., McGuire, S. A., Havlena, J., Gill, P., \& Hershberger, S. L. (2007) Intellectual similarity of virtual twin pairs: Developmental trends. Personality and Individual Differences, 42, 1209-1219.

Segal, N. L., McGuire, S. A., Miller, S., \& Havlena, J. (2008). Tacit coordination in monozygotic twins, dizygotic twins and virtual twins: Effects and implications of genetic relatedness. Personality and Individual Differences, 45, 607-612.

Segal, N. L., McGuire, S. A., \& Stohs, J. (2012). What virtual twins reveal about general intelligence and other behaviors. Personality and Individual Differences, 53, 405-410.

Segal, N. L., \& Montoya, Y. S. (2018). Accidental brothers: The story of twins exchanged at birth and the power of nature and nurture. New York: St Martin's Press.

Segal, N. L., Munson, J. E., Marelich, W. D., Goetz, A. T., \& McGuire, S. A (2013). Meeting of minds: Tacit coordination in monozygotic and dizygotic adolescent and adult twins. Personality and Individual Differences, $58,31-36$.

Segal, N. L., Orozco, E. N., Preston, K. J., \& Gerkens, D. R. (2019). Nonverbal cognition across genetic and environmentally informative kinships: The case for opposite-sex twin pairs and virtual twins. Journal of Experimental Child Psychology, 182, 144-150.

Segal, N. L., Stohs, J. H., \& Evans, K. (2011). Chinese twin children reared apart and reunited: First prospective study of co-twin reunions. Adoption Quarterly, 14, 61-78.

Segal, N. L., Tan, T. X., \& Graham, J. L. (2015). Twins and virtual twins: Do genetic (as well as experiential) factors affect developmental risks? Journal of Experimental Child Psychology, 136, 55-69.

Tan, T. X., Marfo, K., \& Dedrick, R. F. (2010). Early developmental and psychosocial risks and longitudinal behavioral adjustment outcomes for preschool-age girls adopted from China. Journal of Applied Developmental Psychology, 31, 306-314.

Tapp, A. L., Maybery, M. T., \& Whitehouse, A. J. O. (2011). Evaluating the twin testosterone transfer hypothesis: A review of the empirical evidence. Hormones and Behavior, 60, 713-722. 